\title{
To what extent do thermo physical properties of a metallurgical reactor affect the performance of a virtual sensor used for predicting the ledge profile?
}

\author{
Marc LeBreux ${ }^{\mathrm{a}}$, Martin Désilets and Marcel Lacroix \\ Faculté de génie, Université de Sherbrooke, 2500 boul. de l'Université, J1K 2R1, Sherbrooke, Québec, Canada
}

Received 21 February 2014, Accepted 8 January 2016

\begin{abstract}
A non-invasive virtual sensor is employed for the inverse prediction of the time-varying ledge profile that forms inside high-temperature metallurgical reactors filled with a load of phase change material (PCM). The virtual sensor is tested for thermo physical properties of the vessel wall and of the PCM that fall outside the range for which it was originally designed. The results are analyzed and presented in terms of the shift of key thermo physical properties from the reference case. Results indicate that the virtual sensor is more sensitive to the variation of the properties of the phase change material than that of the vessel walls. The virtual sensor response remains accurate for reactor loads of high thermal inertia. The virtual sensor may still be used for reactor loads of low thermal inertia provided that thermo physical properties of the PCM are well-known.
\end{abstract}

Key words: Ledge / metallurgical reactor / virtual sensor / inverse method / unscented Kalman filter / uncertain thermo physical properties

\section{Nomenclature}

\begin{tabular}{|ll|}
\hline \multicolumn{2}{|c|}{ Roman symbols } \\
\hline$C_{p}$ & Specific heat $\left[\mathrm{J}_{\mathrm{kg}}{ }^{-1} \cdot \mathrm{K}^{-1}\right]$ \\
$\mathrm{d} t$ & Time step $[\mathrm{s}]$ \\
$D$ & Space steps $[\mathrm{m}]$ \\
$f$ & Width of the PCM layer $[\mathrm{m}]$ \\
$F$ & Nonlinear function \\
$\mathrm{FDM}$ & Liquid fraction \\
$g$ & Finite-difference model \\
$h$ & Nonlinear function \\
& Heat transfer coefficient imposed \\
$h$ & at the boundary $y=H\left[\mathrm{~W} \cdot \mathrm{m}^{-2} \cdot \mathrm{K}^{-1}\right]$ \\
& Heat transfer coefficient imposed \\
$H$ & at the boundary $x=0\left[\mathrm{~W} \cdot \mathrm{m}^{-2} \cdot \mathrm{K}^{-1}\right]$ \\
$k$ & Height of the brick wall $[\mathrm{m}]$ \\
& Thermal conductivity $\left[\mathrm{W} . \mathrm{m}^{-1} \cdot \mathrm{K}^{-1}\right]$ \\
$k+1 \mid k$ & or sampling instant \\
$k+1 \mid k+1$ & A priori estimate \\
$K$ & A posteriori estimate \\
$L$ & Kalman gain \\
$n$ & Width of the brick wall $[\mathrm{m}]$ \\
$N$ & Length of the state vector \\
\hline
\end{tabular}

a Corresponding author:

marc.lebreux@usherbrooke.ca

\begin{tabular}{|c|c|}
\hline$q^{\prime \prime}$ & Heat flux $\left[\mathrm{W} \cdot \mathrm{m}^{-2}\right]$ \\
\hline$q_{\mathrm{in}}^{\prime \prime}$ & $\begin{array}{l}\text { Input heat flux imposed at the } \\
\text { boundary } x=L+D\left[\mathrm{~W} \cdot \mathrm{m}^{-2}\right]\end{array}$ \\
\hline$P$ & $\begin{array}{l}\text { Covariance matrix of the estimated } \\
\text { state }\end{array}$ \\
\hline$P[0 \mid 0]$ & $\begin{array}{l}\text { Initial covariance matrix of the } \\
\text { estimated state }\end{array}$ \\
\hline$P_{\hat{y}}$ & Innovation covariance \\
\hline$P_{\hat{X} \hat{y}}$ & $\begin{array}{l}\text { Cross covariance between } \hat{X}[k+1 \mid k] \\
\text { and } \hat{y}[k]\end{array}$ \\
\hline $\mathrm{PCM}$ & Phase change material \\
\hline$Q$ & Covariance matrix of the process noise \\
\hline$R$ & $\begin{array}{l}\text { Covariance matrix of the measurement } \\
\text { noise }\end{array}$ \\
\hline$R R M S E$ & Relative root-mean-square error [\%] \\
\hline$\overline{R R M S E}$ & $\begin{array}{l}\text { Mean of the relative root-mean-square } \\
\text { error }[\%]\end{array}$ \\
\hline$s$ & Ledge thickness $[\mathrm{m}]$ \\
\hline$S$ & Ledge profile $[\mathrm{m}]$ \\
\hline$t$ & Time $[s]$ \\
\hline$T$ & Temperature $[\mathrm{K}]$ \\
\hline$u$ & Input signal vector $\left[\mathrm{W} \cdot \mathrm{m}^{-2}, \mathrm{~W} \cdot \mathrm{m}^{-2} \cdot \mathrm{K}^{-1}\right]$ \\
\hline UKF & Unscented Kalman filter \\
\hline
\end{tabular}




\begin{tabular}{|c|c|}
\hline$v$ & Measurement noise $\left[\mathrm{W} . \mathrm{m}^{-2}\right]$ \\
\hline$w$ & Process noise \\
\hline$x$ & State vector or space direction \\
\hline$X$ & Augmented state vector \\
\hline$\hat{X}[0 \mid 0]$ & $\begin{array}{l}\text { Initial augmented state vector } \\
\text { estimate }\end{array}$ \\
\hline$W$ & Scalar weight \\
\hline$y$ & $\begin{array}{l}\text { Space direction or measurement } \\
\text { vector }\left[\mathrm{W} \cdot \mathrm{m}^{-2}, \mathrm{~m}\right]\end{array}$ \\
\hline$\widehat{y}$ & $\begin{array}{l}\text { Predicted output of the state-space } \\
\text { model }\left[\mathrm{W} . \mathrm{m}^{-2}, \mathrm{~m}\right]\end{array}$ \\
\hline$Y_{\text {mes }}$ & Thermal measurements $\left[\mathrm{W} \cdot \mathrm{m}^{-2}, \mathrm{~K}\right]$ \\
\hline$\hat{Y}_{\mathrm{mes}}$ & $\begin{array}{l}\text { Predicted output of the measurements } \\
\text { state-space model }\left[\mathrm{W} . \mathrm{m}^{-2}\right]\end{array}$ \\
\hline & Greek symbols \\
\hline$\delta H$ & Volumetric enthalpy change $\left[\mathrm{J}^{\prime} \mathrm{m}^{-3}\right]$ \\
\hline$\gamma$ & UKF scaling factor \\
\hline$\kappa$ & UKF tuning parameter \\
\hline$\lambda$ & Heat of fusion $\left[{\left.\mathrm{J} . \mathrm{kg}^{-1}\right]}^{-1}\right.$ \\
\hline$\rho$ & Density $\left[\mathrm{kg} \cdot \mathrm{m}^{-3}\right]$ \\
\hline$\sigma_{Q}$ & $\begin{array}{l}\text { Process noise standard deviation } \\
{\left[\mathrm{W} \cdot \mathrm{m}^{-2}, \mathrm{~W} \cdot \mathrm{m}^{-2} \cdot \mathrm{K}^{-1}\right]}\end{array}$ \\
\hline$\sigma_{R}$ & $\begin{array}{l}\text { Measurement noise standard } \\
\text { deviation }\left[\mathrm{W} . \mathrm{m}^{-2}\right]\end{array}$ \\
\hline$\psi[k]$ & Sigma points propagated through $\bar{g}$ \\
\hline$\chi[k]$ & Sigma points \\
\hline \multicolumn{2}{|c|}{$\chi[k+1]$ Sigma points propagated through $\bar{f}$} \\
\hline \multicolumn{2}{|c|}{ Subscripts } \\
\hline$\infty$ & Cooling \\
\hline $\mathrm{amb}$ & Ambient \\
\hline BRICK & Brick wall \\
\hline liquid & Liquid (PCM) \\
\hline PCM & Phase change material \\
\hline solid & Solid (PCM) \\
\hline \multicolumn{2}{|r|}{ Superscripts } \\
\hline - & With augmented state \\
\hline & Estimated \\
\hline$T$ & Transposed matrix \\
\hline
\end{tabular}

\section{Introduction}

An interesting melting/solidification process that occurs within high-temperature metallurgical reactors (Fig. 1), such as electric arc furnaces, blast furnaces and aluminium electrolysis cells, is the formation of protective layers (also called ledges) on the inside surface of the walls. These layers are formed as the molten material comes into contact with the cooled surface of the reactor's wall and undergoes a solidification process. The presence of these layers is desirable as they serve as a protective barrier against the corrosive molten material, thereby maintaining the integrity of the reactor's envelope and prolonging its lifetime [1-3].

Due to the hostile conditions that prevail inside such reactors, it is almost impossible to probe the time-varying shape of the ledges with sensors submerged into the

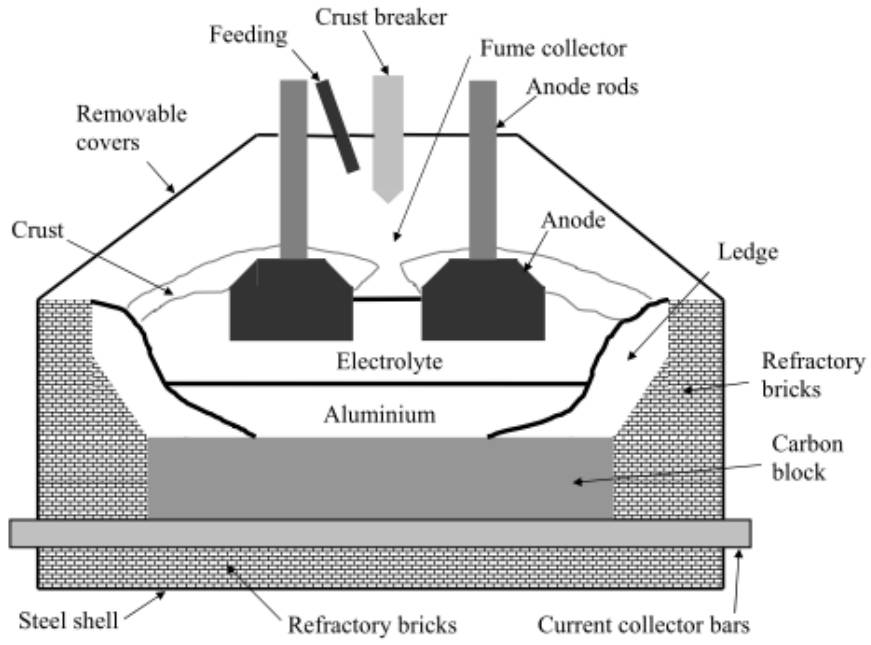

Fig. 1. Cross view of a typical high-temperature metallurgical reactor; an aluminium electrolysis cell with molten material (electrolyte) and its protective ledge.

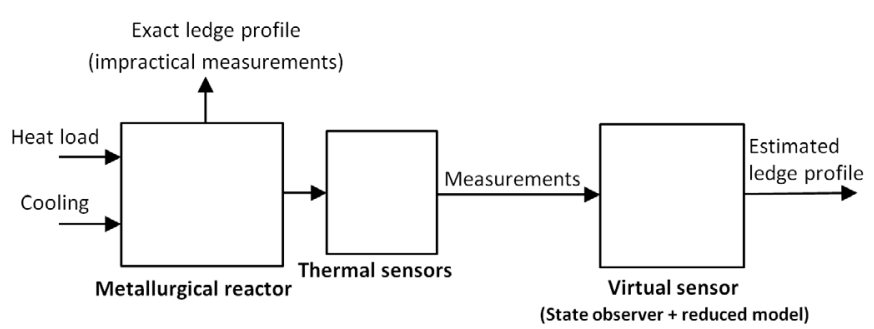

Fig. 2. The virtual sensor comprises a state observer, a reduced model, and sensors located in the reactor's wall [4].

molten material. This is why LeBreux et al. [4] have recently proposed a non-invasive virtual sensor to perform this delicate task. The experimental method relies on data recorded by heat flux sensors embedded into the reactor's wall. The virtual sensor comprises a state observer (a Kalman filter) coupled to a reduced model (a state-space model) of the reactor (Fig. 2). Furthermore, contrary to most geometry estimation methods reported in the open literature [5-11], the virtual sensor is easily adaptable to complex physical phenomena such as phase change processes, transient regimes and nonlinear transfer processes.

In their study, LeBreux et al. [4] assumed that the thermo physical properties of the reactor load and of the walls were well-known. The fact that some of these properties may sometimes be poorly known or that they may be time-varying was ignored. As a result, it is doubtful that the response provided by the proposed virtual sensor would remain accurate under the harsh conditions that prevail inside industrial facilities [12]. The present paper investigates the possibility of using such a virtual sensor in metallurgical reactors for which the thermo physical properties fluctuate or are poorly known.

The effect of erroneous models on the Kalman filter response was examined in the 1960s [13]. In order to reduce the problem associated with erroneous models, adaptive Kalman filtering with unknown state and measurement 
covariance matrices was proposed [14]. More recently, Myers et al. [15] have modified the classic extended Kalman filter algorithm in order to use an adaptive extended Kalman filter for determining the high heat flux spot sources from an array of ultrasonic sensors. However, all of aforementioned studies did not employ the more precise and stable unscented Kalman filter (UKF) algorithm $[4,16,17]$. Recent trends have also shifted towards developing robust versions of such filters in order to handle model uncertainties [18-20]. The theory that underlies robust filtering is more complicated than the Kalman filter algorithms [21]. Few authors have examined the problem of uncertainties with the thermo physical properties and/or with the boundary conditions [22-24]. These analyses were limited solely to linear heat conduction processes. As a result, they cannot be transposed to the present nonlinear melting/solidification problem.

This paper is divided into four sections. First, a twodimensional thermal model of a metallurgical reactor is presented. Second, a virtual sensor for estimating the ledge profile is proposed. Third, the virtual sensor is thoroughly tested for typical operating conditions that prevail inside an industrial facility. Finally, the effect of modifying thermo physical properties on the performance of the virtual sensor is investigated.

\section{Thermal model of the reactor}

The 2-D phase change problem under investigation is depicted in Figure 3. A time-varying ledge profile $s(y, t)$, which corresponds to the phase front location, is pressed against the inner surface of a brick wall of thickness $L$ and height $H$. The outer surface of the brick wall is cooled by forced convection heat transfer with an impinging air jet of constant temperature $T_{\mathrm{amb}}$ and with a heat transfer coefficient $\bar{h}$, calculated from established correlations [25]. A time-varying uniform heat flux $q_{\text {in }}^{\prime \prime}(t)$, which represents the heat load supplied to the reactor, is imposed at the right boundary condition at $x=L+D$. The top of the phase change material (PCM) is cooled by forced convection with an air stream at constant temperature $T_{\infty}$ and a time-varying uniform heat transfer coefficient $h(t)$. These heat losses reflect the normal operating conditions and disturbances that prevail in metallurgical processes [26-28].

The mathematical model for the phase change problem rests on the following assumptions:

- The finite-difference model (FDM) mimics the thermal behavior of a real metallurgical reactor $[29,30]$.

- The phase change problem is non-isothermal. A mushy zone thus separates the solidus $T_{\text {solidus }}$ from the liquidus $T_{\text {liquidus }}$.

- The thermal contact resistance between the brick wall and the ledge is ignored. Its effect may however be taken into account by modifying the thermal conductivities of neighbouring materials.

- The thermal properties of the PCM are temperature independent; they may, however, be different for the liquid and the solid phases.

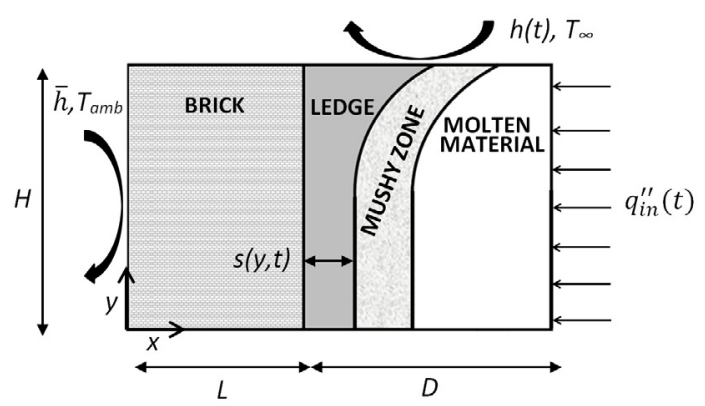

Fig. 3. Schematic of the direct phase change problem inside a high-temperature metallurgical reactor (ledge = solid PCM, molten material $=$ liquid $\mathrm{PCM}$ ).

- The heat transfer across the liquid layer of the PCM is convection dominated. The effect of the flow circulation in the liquid layer may however be accounted by means of an "augmented effective thermal conductivity" [31].

The governing heat diffusion equation for the wall and the PCM are then stated as:

$$
\rho C_{p} \frac{\partial T}{\partial t}=\nabla \cdot(k \nabla T)-\delta H \frac{\partial F}{\partial t}
$$

The boundary conditions are:

$$
\begin{aligned}
\left.k \frac{\partial T}{\partial y}\right|_{x, y=0} & =0 \\
\left.k \frac{\partial T}{\partial x}\right|_{x=0, y} & =\bar{h}\left[T(x=0, y)-T_{\mathrm{amb}}\right] \\
\left.k \frac{\partial T}{\partial y}\right|_{x<L, y=H} & =0 \\
\left.k \frac{\partial T}{\partial y}\right|_{x \geqslant L, y=H} & =h(t)\left[T(x, y=H)-T_{\infty}\right] \\
\left.k \frac{\partial T}{\partial x}\right|_{x=L+D, y} & =q_{\mathrm{in}}^{\prime \prime}(t)
\end{aligned}
$$

and the initial condition is:

$$
T(x, y, t=0)=T_{0}(x, y)
$$

The volumetric enthalpy change $\delta H$ is defined as $\delta H=$ $\rho\left(C_{p \text {, liquid }}-C_{p \text {,solid }}\right) T+\rho \lambda$. The liquid fraction $F$ varies linearly between the solidus temperature $T_{\text {solidus }}$ and the liquidus temperature $T_{\text {liquidus }}$ in the following manner:

$$
\begin{aligned}
F & =F(T) \\
& =\left\{\begin{array}{cl}
0 & T \leqslant T_{\text {solidus }} \\
\left(\frac{T-T_{\text {solidus }}}{T_{\text {liquidus }}-T_{\text {solidus }}}\right) & T_{\text {solidus }}<T<T_{\text {liquidus }} \\
1 & T \geqslant T_{\text {liquidus }}
\end{array}\right.
\end{aligned}
$$

Equations (1) and (8) were discretized using second-order finite differences in space, and first-order differences in 
Table 1. Thermo physical properties of the brick wall and of the PCM for a typical high-temperature metallurgical reactor $[29,30]$.

\begin{tabular}{|c|c|}
\hline Parameter & Value \\
\hline$C_{p, \text { BRICK }}\left(\mathrm{J} . \mathrm{kg} \cdot \mathrm{K}^{-1}\right)$ & 875 \\
\hline$\rho_{\text {BRICK }}\left(\mathrm{kg} . \mathrm{m}^{-3}\right)$ & 2600 \\
\hline$k_{\mathrm{BRICK}}\left(\mathrm{W} \cdot \mathrm{m} \cdot \mathrm{K}^{-1}\right)$ & 16.8 \\
\hline$C_{p, \mathrm{PCM}, \text { solid }}\left(\mathrm{J} . \mathrm{kg} . \mathrm{K}^{-1}\right)$ & 1800 \\
\hline$C_{p, \mathrm{PCM}, \text { liquid }}\left(\mathrm{J} \cdot \mathrm{kg} \cdot \mathrm{K}^{-1}\right)$ & 1800 \\
\hline$\rho_{\mathrm{PCM}}\left(\mathrm{kg} \cdot \mathrm{m}^{-3}\right)$ & 2100 \\
\hline$k_{\mathrm{PCM}, \text { solid }}\left(\mathrm{W} \cdot \mathrm{m} \cdot \mathrm{K}^{-1}\right)$ & 1 \\
\hline$k_{\mathrm{PCM}, \text { liquid }}\left(\mathrm{W} \cdot \mathrm{m} \cdot \mathrm{K}^{-1}\right)$ & 10 \\
\hline$\lambda_{\mathrm{PCM}}\left({\left.\mathrm{J} . \mathrm{kg}^{-1}\right)}^{-1}\right.$ & $5.1 \times 10^{5}$ \\
\hline$T_{\text {solidus }}(\mathrm{K})$ & 1213 \\
\hline$T_{\text {liquidus }}(\mathrm{K})$ & 1233 \\
\hline$T_{\mathrm{amb}}(\mathrm{K})$ & 303 \\
\hline$T_{\infty}(\mathrm{K})$ & 433 \\
\hline $\bar{h}\left(\mathrm{~W} \cdot \mathrm{m}^{-2} \cdot \mathrm{K}^{-1}\right)$ & 30 \\
\hline
\end{tabular}

time with an implicit scheme. The resulting set of algebraic equations was solved with a Gauss-Seidel algorithm. The liquid fraction $F$ in equation (1) is determined according to the enthalpy method, an iterative procedure developed by Voller and Swaminathan [32].

The above finite-difference model (FDM), equations (1)-(8) defined here as the exact model of the metallurgical reactor, was thoroughly tested and validated with analytical solutions and results reported in the open literature [32-34]. For more details on the validation of the numerical model, the reader is referred to reference [4].

The physical properties of the brick wall and of the PCM for a typical high-temperature metallurgical reactor are presented in Table 1 [29,30]. From these references, the thickness and height of the brick wall were fixed at $L=0.1 \mathrm{~m}$ and $H=0.2 \mathrm{~m}$ respectively. The initial width of the PCM layer (solid, mushy, and liquid) was set equal to $D=0.1 \mathrm{~m}$ (Fig. 3). As for the time step $\mathrm{d} t$ and the mesh size $\mathrm{d} x$ and $\mathrm{d} y$, they were set to $100 \mathrm{~s}, 4 \times 10^{-3} \mathrm{~m}$ and $4 \times 10^{-3} \mathrm{~m}$ respectively. Simulations carried out with these numerical parameters proved to be a good compromise between numerical accuracy and CPU time requirements. The FDM provides the evolution of the temperature field $T(x, y, t)$, the heat flux distribution $q^{\prime \prime}(x, y, t)$, and the ledge profile $s(y, t)$.

\section{Virtual sensor}

In the inverse problem, the input heat flux $q_{\text {in }}^{\prime \prime}(t)$ and the heat transfer coefficient $h(t)$ are unknown. Consequently, the ledge profile $S(t)=s(y, t)$ cannot be estimated directly (Fig. 4). However, from thermal measurements $Y_{\text {mes }}(x, y, t)$ provided by sensors embedded into the reactor's wall, it is possible to estimate $q_{\text {in }}^{\prime \prime}(t)$ and $h(t)$

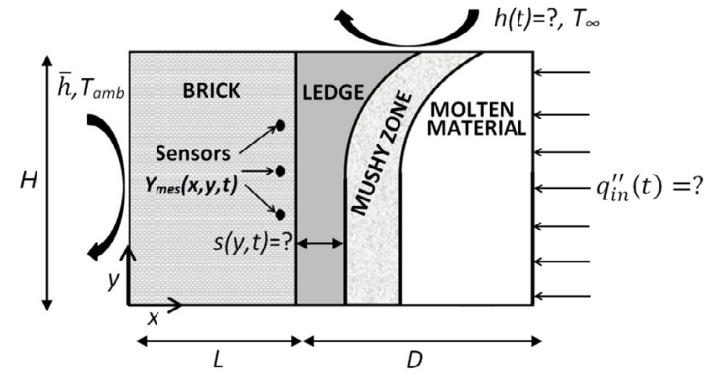

Fig. 4. Schematic of the phase change inverse problem inside a high-temperature metallurgical reactor.

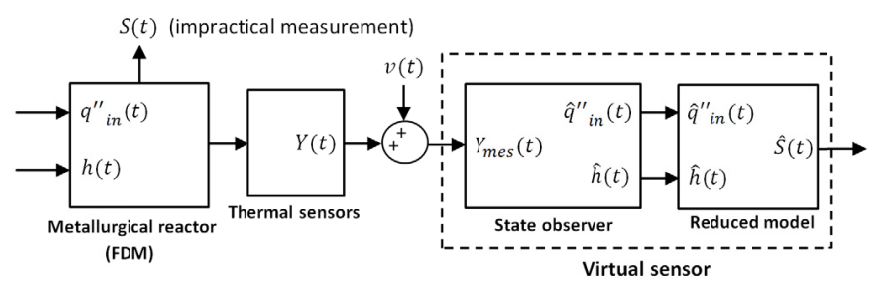

Fig. 5. The virtual sensor for estimating the ledge profile; a state observer coupled to a reduced model.

with a state observer and therefore to predict the timevarying ledge profile $\hat{S}(t)$ using the reduced model of the metallurgical reactor. The overall inverse methodology is summarized schematically in Figure 5.

The inverse method is equivalent to a virtual sensor that comprises an estimation algorithm (a state observer and a reduced model) fed with measurements taken by sensors $\left(Y_{\text {mes }}\right)$ that allow on-line estimation of the process variables. In the present case, the process variable is the ledge profile $\hat{S}$. The mathematical formulation of the virtual sensor is detailed in Appendix A.

First, a reference case is defined for the virtual sensor. The virtual sensor knows exactly the thermo physical properties of the metallurgical reactor (Table 1 ). The observed behaviour of the metallurgical reactor is equivalent to that of its reduced model [4]. Also, the tuning factor of the virtual sensor, represented by the covariance matrices $Q$ and $R$ (Table 2 ), are chosen so as to reach a compromise between the stability and the tracking capability of the inverse method. The stability refers to the oscillations in the solution triggered by the measurement noise. The tracking capability refers to the time lag value that results from the heat diffusion phenomenon. The choice of these matrices is akin to choosing the correct number of future steps in Beck's function specification method [35] and akin to choosing the convenient regularization parameter in Tikhonov's regularization technique [36].

The virtual sensor for the reference case was thoroughly tested for estimating the time-varying input heat flux $q_{\text {in }}^{\prime \prime}$ and the heat transfer coefficient $h$, and therefore for predicting the time-varying profile of the ledge $\hat{S}$ inside the reactor. For this reason, numerical simulations were carried out for typical operating conditions that prevail in such facilities [25-28]. 
Table 2. Covariance matrices of the virtual sensor.

\begin{tabular}{cc}
\hline Covariance matrices & Values \\
\hline$Q$ & {$\left[\begin{array}{ccc}0^{2} & 0 & 0 \\
0 & 0.005^{2} & 0 \\
0 & 0 & 0.01^{2}\end{array}\right]$} \\
\hline$R$ & {$\left[\begin{array}{cc}100^{2} & 0 \\
0 & 100^{2}\end{array}\right]$} \\
\hline
\end{tabular}

Figure 6a compares the corresponding unknown conditions $\left(q_{\mathrm{in}}^{\prime \prime}, h\right)$ estimated by the state observer to the exact conditions imposed at the boundaries of the metallurgical reactor. Results indicate that with only two heat flux sensors located at $x=L, y=1 / 4 H$ and $x=L, y=3 / 4 H$, the state observer is able to predict the unknown boundary conditions. One can still note the time lag between the exact and estimated boundary conditions. This is a consequence of using thermal measurements that are affected by the heat diffusion phenomenon.

Using these estimated conditions, the virtual sensor can then predict the ledge profile. The corresponding time-varying exact $S$ and estimated $\hat{S}$ ledge profiles are compared in Figure 6b.

To characterize the accuracy of the virtual sensor prediction, the relative root-mean-square error $(R R M S E)$ is invoked (Eq. (9)). The RRMSE represents, at any time, the discrepancy between the exact and the estimated ledge profiles (Fig. 6c)

$$
\operatorname{RRMSE}(t)=\sqrt{\frac{1}{\left(y_{2}-y_{1}\right)} \sum_{y=y_{1}}^{y_{2}}\left(\frac{s(y, t)-\hat{s}(y, t)}{s(y, t)}\right)^{2}}
$$

$s$ and $\hat{s}$ are the exact and the predicted ledge thicknesses respectively. $y_{1}=1 / 6 H$ and $y_{2}=5 / 6 H$.

By comparing the results from Figures $6 \mathrm{~b}$ and $6 \mathrm{c}$, one may observe that the accuracy of the ledge profile prediction is strongly affected by the thermal resistance across the ledge. As the ledge thickness increases, the thermal resistance increases and the time lags are prolonged, thereby affecting the accuracy of the estimated ledge profile. However, one must bear in mind that the experimental uncertainty associated to the standard manual ledge thickness measurement can be in the range of $\pm 10-20 \%$, and in order to perform the measurements, the cover needs to be open, thus releasing to the surrounding environment a large quantity of heat and chemicals. Moreover, this propensity of the virtual sensor to provide fast and increasingly accurate predictions of the ledge thickness as it shrinks is of utmost interest for the operator. Indeed, it is precisely when the ledge becomes thin and threatens to vanish that it is more than ever imperative for the control system to react promptly and accurately so as to prevent damages to the facility.
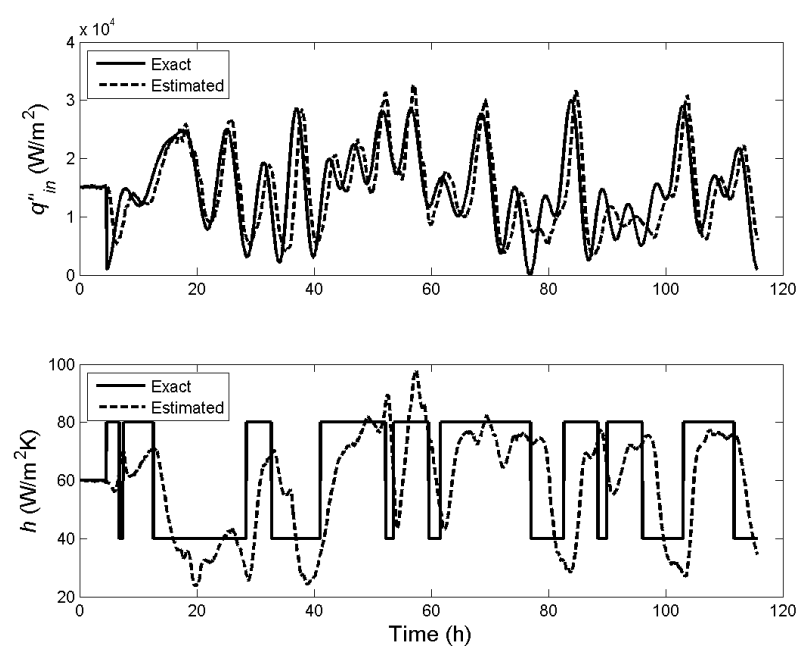

(a)
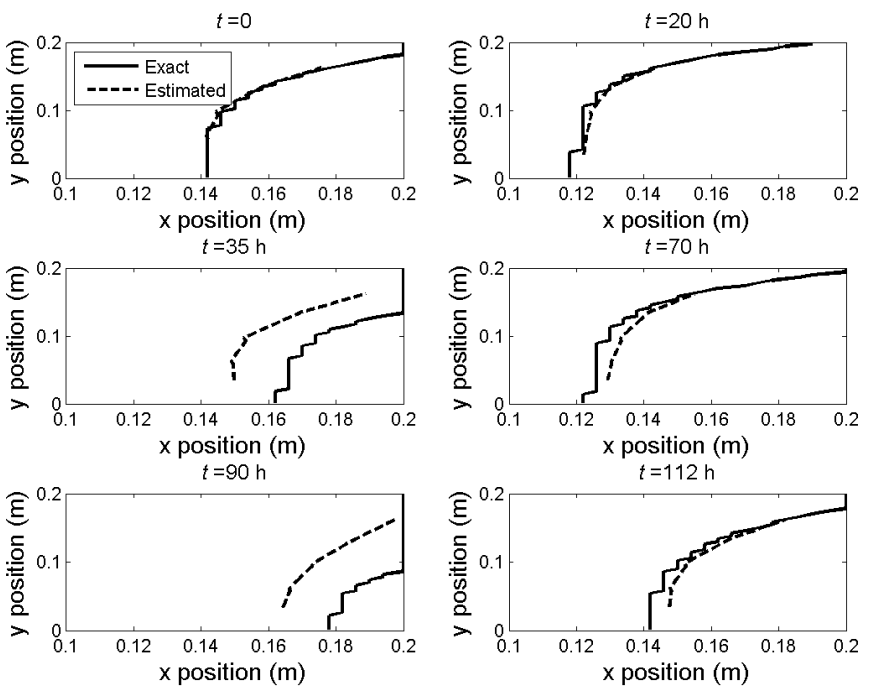

(b)

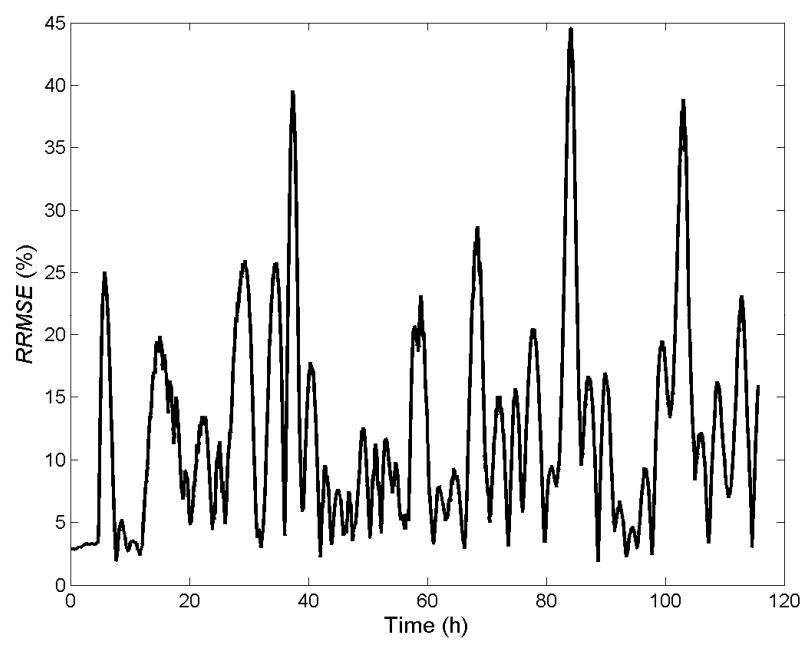

(c)

Fig. 6. (a) Exact and estimated input heat flux $q_{\text {in }}^{\prime \prime}$ and heat transfer coefficient $h$ - Reference case. (b) Exact and estimated ledge profile - Reference case. (c) Ledge profile estimation RRMSE - Reference case. 


\section{Effect of the thermo physical properties on the performance of the virtual sensor}

The purpose of this section is to determine the performance of the virtual sensor when it is employed in a reactor for which the thermo physical properties of the materials fluctuate and/or are poorly known. The problem is depicted in Figure 7. The thermo physical properties of the metallurgical reactor differ from those of the reference case. As a result, the thermal behaviour of the reactor will differ from that of the reduced model for the reference case, thereby challenging the virtual sensor.

The results of the simulations are analysed and presented in terms of a parameter $\varepsilon$. $\varepsilon$ represents the shift of the thermo physical property from the reference case (Table 1). The shifted property is obtained simply by multiplying the property of the reference case by $(1+\varepsilon / 100)$. Note that $\varepsilon$ is expressed here in terms of percentage. Thus, the thermo physical property is overestimated for $\varepsilon>0$ and underestimated for $\varepsilon<0$. Figures 8 and 9 assess the performance of the virtual sensor with the average $\overline{R R M S E}$ defined as:

$$
\overline{R R M S E}=\frac{1}{N} \sum_{t=0}^{N} R R M S E(t)
$$

The $\overline{R R M S E}$ represents the discrepancy between the exact and the estimated ledge profiles integrated over time.

Examination of Figure 8 reveals that for both sensible and latent properties, the inverse predictions of the ledge profiles worsen when the thermo physical properties are underestimated $(\varepsilon<0)$. The response of the virtual sensor improves however when the thermo physical properties are overestimated $(\varepsilon>0)$. These findings illustrate the fact that the virtual sensor can capture relatively well the slow dynamics and the small variations in the ledge thickness for reactor loads of high thermal inertia $(\varepsilon>0)$. On the other hand, the response of the virtual sensor deteriorates for reactor load of low thermal inertia $(\varepsilon<0)$. In this case, the ledge thickness varies more rapidly and with greater amplitude. Note that the thermo physical properties of the PCM, i.e., $k_{\mathrm{PCM}}, \rho_{\mathrm{PCM}}, C_{p, \mathrm{PCM}}, T_{\text {solidus }}$, and $\lambda_{\mathrm{PCM}}$ all reflect the inertia of the system. Consequently, the thermal inertia of the reactor is proportional to $\varepsilon$. For $\varepsilon>0$, the thermal inertia is overestimated. For $\varepsilon<0$, it is underestimated.

The virtual sensor may still be employed for the prediction of the ledge thickness in reactors with low thermal inertia loads. In these cases however, the thermo physical properties of the load, particularly its heat capacity, must be precisely known.

Figure 9 shows that the predictions of the ledge profile worsen when the thermo physical properties of the vessel walls are overestimated $(\varepsilon>0)$. It gets even worse when they are underestimated $(\varepsilon<0)$. It shows, once again, that the virtual sensor is suited for reactor loads of high thermal inertia. Also, the virtual sensor is more robust, that is it can better cope with the uncertainty in the brick wall properties. The variation of its $\overline{R R M S E}$ is smaller than that for the PCM (Fig. 8).

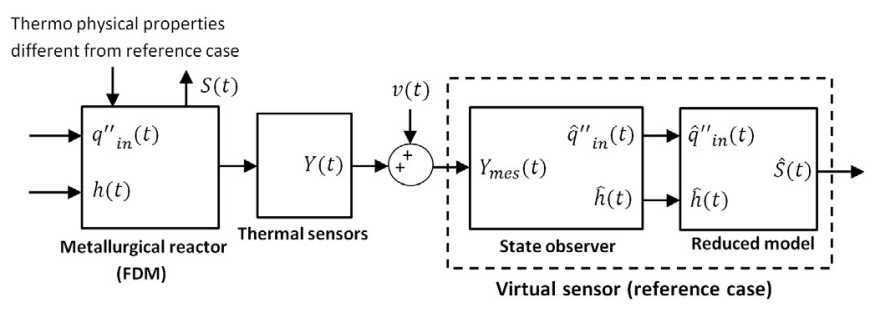

Fig. 7. Does the vitual sensor estimating the ledge profile remain accurate when the thermo physical properties of the metallurgical reactor are different from the reference case?

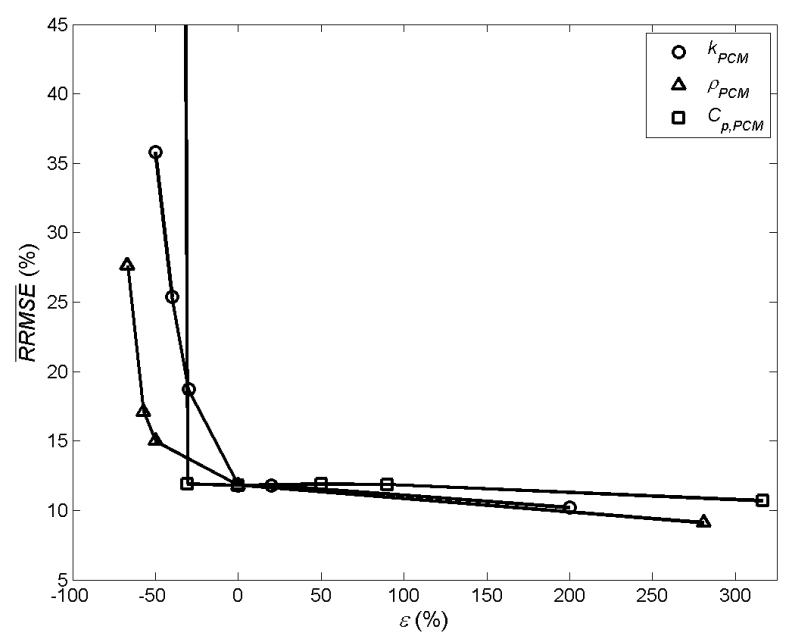

(a)

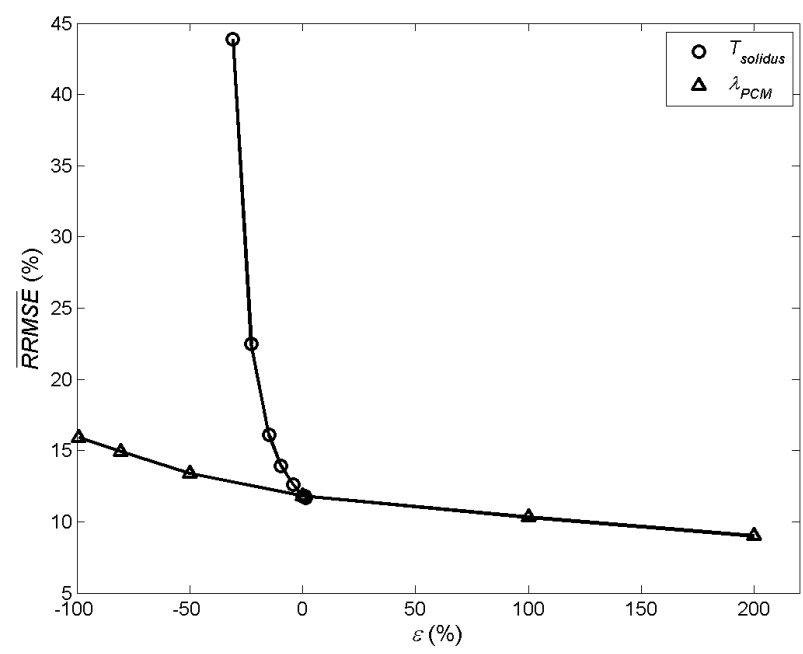

(b)

Fig. 8. The ledge profile estimation $\overline{R R M S E}$ for different value of $\varepsilon-$ PCM. (a) Sensible properties. (b) Latent properties.

\section{Concluding remarks}

A non-invasive virtual sensor was employed for the inverse prediction of the time-varying ledge profile that forms inside high-temperature metallurgical reactors filled with a load of phase change material (PCM). The virtual sensor was tested for thermo physical properties of the vessel walls and of the PCM that fall outside the 


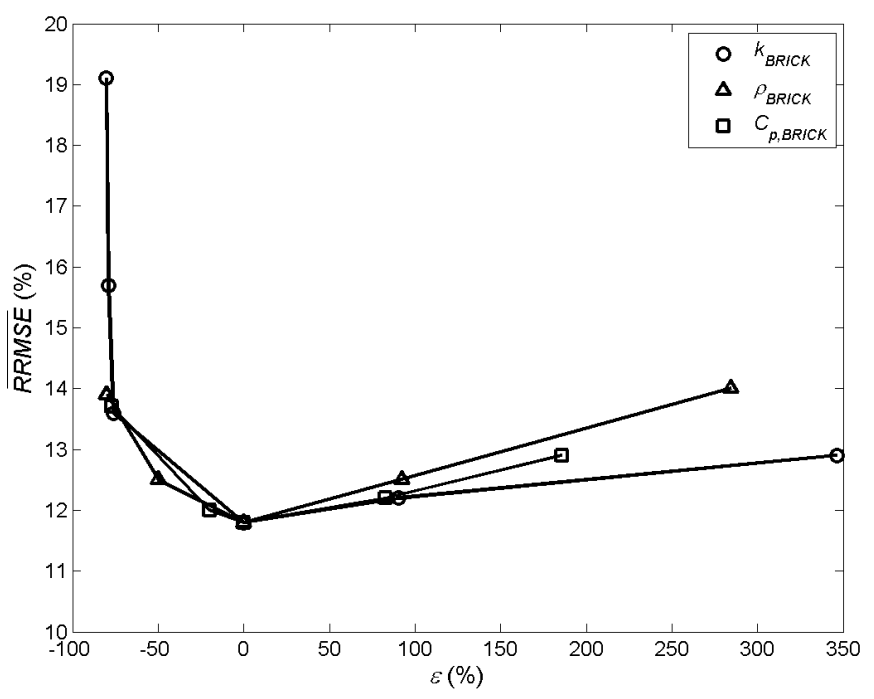

Fig. 9. The ledge profile estimation $\overline{R R M S E}$ for different value of $\varepsilon-$ Brick.

range for which it was originally designed. The results indicate that the virtual sensor is more sensitive to the shift of the properties of the phase change material than that of the vessel walls. The virtual sensor response remains accurate for reactor loads of high thermal inertia. The virtual sensor may still be used for reactor loads of low thermal inertia provided that thermo physical properties of the PCM are well-known.

In order to improve the robustness of the present virtual sensor in cases for which the thermo physical properties are poorly known, a $H_{\infty}$ version of the Kalman filter is currently developed. This approach will allow the integration of an uncertainty model into the filter design and, as a result, it will provide a more stable filter. Results of this study will be reported in the open literature later.

Acknowledgements. The authors are very grateful to the Natural Sciences and Engineering Research Council of Canada (NSERC) for their financial support.

\section{Appendix A : Virtual sensor algorithm}

This appendix explains how that the virtual sensor predicts the time-varying ledge profile $\hat{S}$ of a metallurgical reactor from thermal measurements $Y_{\text {mes }}$ of sensors embedded into the reactor wall.

The reduced model of the metallurgical reactor is defined by the combination of two nonlinear state-space models (NSSM) represented in the following discrete form:

$$
\begin{aligned}
& x[k+1]=f(x[k], u[k])+w[k] \\
& y[k]=g(x[k])+v[k]
\end{aligned}
$$

where $k$ corresponds to the sampling instant. $f$ and $g$ are nonlinear functions. $y$ is the measurement vector, that is the thermal measurements $Y_{\text {mes }}$ for the first NSSM and the ledge profile $S$ for the second NSSM. $u=\left[\begin{array}{ll}q_{\text {in }}^{\prime \prime} & h\end{array}\right]^{T}$ is the input vector equivalent to the time-varying boundary conditions (Eqs. (5) and (6)). $v$ is the sensor noise. It is considered as a zero mean Gaussian white noise with covariance $R$ defined by the covariance matrix of the measurement noise (Eq. (A.3)). $w$ is the process noise. It accounts for the disturbances to the metallurgical reactor dynamics. It is also assumed to be a zero mean Gaussian white noise with covariance $Q$ defined as the covariance matrix of the process noise (Eq. (A.4)).

$$
\begin{aligned}
& v[k] \sim N(0, R)=N\left(0, \sigma_{R}^{2}\right) \\
& w[k] \sim N(0, Q)=N\left(0, \sigma_{Q}^{2}\right)
\end{aligned}
$$

In order to estimate the unknown input heat flux $q_{\text {in }}^{\prime \prime}$ and the heat transfer coefficient $h$ from the thermal measurements $Y_{\text {mes }}$, a state observer approach is employed. This consists of a set of mathematical equations that provide an efficient recursive mean to estimate the unknown state of a process (the state vector). In order to estimate correctly the process state, the state observer proceeds through two steps: first, the time update step (prediction) which provides an initial estimate of the state vector from the knowledge of the process dynamic model; second, the measurement update step (correction) which integrates sensors measurements in order to refine the state vector initial estimate.

In order to estimate the unknown input heat flux $q_{\text {in }}^{\prime \prime}$ and the heat transfer coefficient $h$ from the thermal measurements $Y_{\text {mes }}$, the state observer is employed with an augmented state where the augmented state corresponds to the unknown input heat flux $q_{\text {in }}^{\prime \prime}$ and heat transfer coefficient $h$. Equations (A.1) and (A.2) can thus be modified using the following new state vector defined as $X=\left[\begin{array}{lll}x & q_{\text {in }}^{\prime \prime} & h\end{array}\right]^{T} . x$ corresponds to the state vector of the reduced model (first NSSM), and $\bar{f}$ and $\bar{g}$ are nonlinear functions with augmented state (Eqs. (A.5) and (A.6)).

$$
\begin{aligned}
& X[k+1]=\bar{f}(X[k])+w[k] \\
& y[k]=\bar{g}(X[k])+v[k]
\end{aligned}
$$

The objective of the state observer, an unscented Kalman filter (UKF) in the current work, is to estimate the state vector $X$, defined as $\hat{X}$, given the process dynamic model (Eqs. (A.5) and (A.6)) and the thermal measurements $Y_{\text {mes. }}$. Due to the fact that it is easier for the UFK to approximate a probability distribution, i.e., an arbitrary nonlinear function, the sigma points are selected before the prediction/correction steps are undertaken [17]. The sigma points are chosen so that their mean and covariance correspond exactly to $\hat{X}$ and $P$. The error covariance matrix $P$ characterizes the accuracy of the estimated state vector $\hat{X}$. Each sigma point is then propagated through the nonlinear functions in order to obtain a cloud of transformed points. The new estimated mean and covariance are then computed based on their statistics. This numerical procedure is called unscented transformation. The unscented transformation is a method for calculating the 


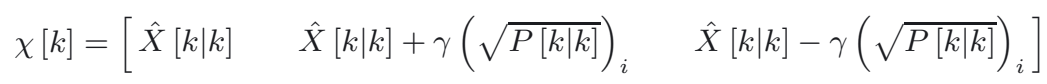

statistics of a random variable which undergoes a nonlinear transformation [17].

Let $\chi[k]$ be defined as a set of $2 n+1$ sigma points where $n$ corresponds to the length of the augmented state vector $X$

$$
\text { see equation (A.7) above }
$$

$\gamma=\sqrt{n+\kappa}$ is a scaling factor that determines the spread of the sigma points around $\hat{X}[k \mid k]$. $\kappa$ was chosen to be in accordance with the heuristic $n+\kappa=3[37] .(\sqrt{P[k \mid k]})_{i}$ is the $i$ th column of the matrix square-root of the error covariance matrix $P[k \mid k]$. It is calculated using Cholesky decomposition [38]. The sigma points are then propagated through the nonlinear function $\bar{f}$ (Eq. (A.8)).

$$
\chi[k+1]=\bar{f}(\chi[k])
$$

The mean and covariance of $\chi[k+1]$ are approximated using a weighted sample mean and covariance (Eqs. (A.9) and (A.10)) in the time update step (prediction).

$$
\begin{aligned}
\hat{X}[k+1 \mid k] & =\sum_{i=0}^{2 n} W_{i} \chi_{i}[k+1] \\
P[k+1 \mid k]= & \sum_{i=0}^{2 n}\left\{W_{i}\left(\chi_{i}[k+1]-\hat{X}[k+1 \mid k]\right)\right. \\
& \left.\times\left(\chi_{i}[k+1]-\hat{X}[k+1 \mid k]\right)^{T}\right\}+Q
\end{aligned}
$$

$\hat{X}[k+1 \mid k]$ and $P[k+1 \mid k]$ are the a priori state vector estimate and error covariance for the next time step respectively. $\chi_{i}[k+1]$ corresponds to the $i$-th column of $\chi[k+1] . W_{i}$ are scalar weights (Eqs. (A.11) and (A.12)).

$$
\begin{aligned}
W_{0} & =\frac{\kappa}{(n+\kappa)} \\
W_{i} & =\frac{1}{2(n+\kappa)} \quad \text { for } \quad i=1,2, \ldots, 2 n
\end{aligned}
$$

The sigma points are now propagated through the nonlinear function $\bar{g}$ (Eq. (A.13))

$$
\psi[k]=\bar{g}(\chi[k+1])
$$

Once again, the mean and covariance of $\psi[k]$ are approximated with a weighted sample mean and covariance (Eqs. (A.14) and (A.15)). $\psi_{i}[k]$ is the $i$ th column of $\psi[k]$.

$$
\begin{aligned}
& \hat{y}[k]=\sum_{i=0}^{2 n} W_{i} \psi_{i}[k] \\
& P_{\hat{y}}[k]=\sum_{i=0}^{2 n}\left\{W_{i}\left(\psi_{i}[k]-\hat{y}[k]\right)\left(\psi_{i}[k]-\hat{y}[k]\right)^{T}\right\}+R
\end{aligned}
$$

$\hat{y}[k]$ and $P_{\hat{y}}[k]$ are the measurement prediction and innovation covariance respectively. As for the cross covariance between $\hat{X}[k+1 \mid k]$ and $\hat{y}[k]$, it is shown in equation (A.16),

$$
\begin{aligned}
& P_{\hat{X} \hat{y}}[k]= \\
& \sum_{i=0}^{2 n}\left\{W_{i}\left(\chi_{i}[k+1]-\hat{X}[k+1 \mid k]\right)\left(\psi_{i}[k]-\hat{y}[k]\right)^{T}\right\}
\end{aligned}
$$

The measurement update step (correction) can then integrate a new measurement $Y_{\text {mes }}$ into the a priori estimates $\hat{X}[k+1 \mid k]$ and $P[k+1 \mid k]$ in order to obtain an improved a posteriori estimate for $\hat{X}[k+1 \mid k+1]$ and $P[k+1 \mid k+1]$ (Eqs. (A.17) and (A.18)). The unscented Kalman gain $K$ is given in equation (A.19).

$$
\begin{aligned}
& \hat{X}[k+1 \mid k+1]=\hat{X}[k+1 \mid k]+K[k]\left(Y_{\text {mes }}[k]-\hat{y}[k]\right) \\
& P[k+1 \mid k+1]=P[k+1 \mid k]-K[k] P_{\hat{y}}[k] K[k]^{T} \\
& K[k]=P_{\hat{X} \hat{y}}[k]\left(P_{\hat{y}}[k]\right)^{-1}
\end{aligned}
$$

From the unknown input heat flux $\hat{q}_{\text {in }}^{\prime \prime}$ and heat transfer coefficient $\hat{h}$ provided by the state observer, the ledge profile can be calculated with the reduced model of the metallurgical reactor (second NSSM, Eqs. (A.1) and (A.2)).

$$
\begin{aligned}
x[k+1] & =f(x[k], \hat{u}[k]) \\
y[k] & =g(x[k])
\end{aligned}
$$

$\hat{u}=\left[\begin{array}{ll}\hat{q}_{\text {in }}^{\prime \prime} & \hat{h}\end{array}\right]^{T}$ is a new input vector provided by the state observer, and $y=\hat{S}$ is the estimated ledge profile.

\section{References}

[1] M.P. Taylor, B.J. Welch, M.J. O'Sullivan, Sidewall Ledge Dynamics in Cells Used for Electrowinning Aluminium, Proceedings of the Eleventh Australian Conference on Chemical Engineering, 1983, pp. 493-500.

[2] J.G. Peacey, G.W. Medlin, Cell Sidewall Studies at Noranda Aluminium, Proceedings of the Minerals, Metals \& Materials Society (TMS), TMS, Warrendale, PA, 1979, pp. $475-491$.

[3] J. Brännbacka, H. Saxén, Model for Fast Computation of Blast Furnace Hearth Erosion and Buildup Profiles, Ind. Eng. Chem. Res. 47 (2008) 7793-7801.

[4] M. LeBreux, M. Désilets, M. Lacroix, Prediction of the Time-Varying Ledge Profile inside a High-Temperature Metallurgical Reactor with an Unscented Kalman Filter Based Virtual Sensor, Numer. Heat Transfer A 64 (2013) $551-576$. 
[5] C.K. Chen, C.R. Su, Inverse Estimation for Temperatures of Outer Surface and Geometry of Inner Surface of Furnace with Two Layer walls, Energy Convers. Manage. 49 (2008) 301-310.

[6] C.R. Su, C.K. Chen, Geometry Estimation of the Furnace Inner Wall by an Inverse Approach, Int. J. Heat Mass Transfer 50 (2007) 3767-3773.

[7] C.R. Su, C.K. Chen, W.L. Liu, H.Y. Lai, Estimation for Inner Surface Geometry of Furnace Wall Using Inverse Process Combined with Grey Prediction Model, Int. J. Heat Mass Transfer 52 (2009) 3595-3605.

[8] J. Torrkulla, H. Saxén, Model of the State of the Blast Furnace Hearth, ISIJ Int. 40 (2000) 438-447.

[9] M. Gonzalez, M.B. Goldschmit, Inverse Geometry Heat Transfer Problem Based on a Radial Basis Functions Geometry Representation, Int. J. Numer. Methods Eng. 65 (2006) 1243-1268.

[10] C.H. Huang, M.T. Chaing, A Transient ThreeDimensional Inverse Geometry Problem in Estimating the Space and Time-Dependent Irregular Boundary Shapes, Int. J. Heat Mass Transfer 51 (2008) 5238-5246.

[11] D.P. Baker, G.S. Dulikravich, B.H. Dennis, T.J. Martin, Inverse Determination of Eroded Smelter Wall Thickness Variation Using an Elastic Membrane Concept, ASME, J. Heat Transfer 132 (2010) 052101-1-052101-8.

[12] M. Kano, M. Ogawa, The State of the Art in Chemical Process Control in Japan: Good Practice and Questionnaire Survey, J. Process Control 20 (2010) 969982.

[13] H. Heffes, The Effect of Erroneous Models on the Kalman Filter Response, IEEE Trans. Automat. Control 11 (1966) 541-543.

[14] R. Mehra, On the Identification of Variances and Adaptive Kalman Filtering, IEEE Trans. Automat. Control 15 (1970) 175-184.

[15] M.R. Myers, A.B. Jorge, D.E. Yuhas, D.G. Walker, An Adaptive Extended Kalman Filter Incorporating State Model Uncertainty for Localizing a High Heat Flux Spot Source Using an Ultrasonic Sensor Array, Comput. Model. Eng. Sci. 83 (2012) 221-248.

[16] M. LeBreux, M. Désilets, M. Lacroix, An Unscented Kalman Filter Inverse Heat Transfer Method for the Prediction of the Ledge Thickness Inside HighTemperature Metallurgical Reactors, Int. J. Heat Mass Transfer 57 (2013) 265-273.

[17] S.J. Julier, J.K. Ulhmann, Unscented filtering and nonlinear estimation, Proc. IEEE 92 (2004) 401-422.

[18] L. Xie, Y. Soh, C.E. de Souza, Robust Kalman Filtering for Uncertain Discrete-Time Systems, IEEE Trans. Automat. Control 39 (1994) 1310-1314.

[19] A.V. Savkin, I.R. Petersen, Robust State Estimation and Model Validation for Discrete-Time Uncertain Uncertain Systems with a Deterministic Description of Noise and Uncertainty, Automatica 34 (1998) 271-274

[20] F. Wang, V. Balakrishnan, Robust Kalman Filters for Linear Time-Varying Systems with Stochastic Parametric Uncertainties, IEEE Trans. Signal Process. 50 (2002) 803-813.

[21] D. Simon, Optimal state estimation: Kalman, $H_{\infty}$ and nonlinear approaches, Wiley-Interscience, Hoboken, 2006.
[22] B. Blackwell, J.V. Beck, A Technique for Uncertainty Analysis for Inverse Heat Conduction Problems, Int. J. Heat Mass Transfer 53 (2010) 753-759.

[23] A.F. Emery, A.V. Nenarokomov, T.D. Fadale, Uncertainties in Parameter Estimation: the Optimal Experiment Design, Int. J. Heat Mass Transfer 43 (2000) 3331-3339.

[24] S.E. Davis, N.T. Wright, Optimal Positioning of Temperature Measurements to Estimate Thermal Diffusivity, Int. J. Thermophys. 34 (2013) 1021-1038.

[25] F.P. Incropera, D.P. DeWitt, Fundamentals of Heat and Mass Transfer, 5th edition, John Wiley \& Sons, 2002, pp. $428-434$

[26] M.P. Taylor, G.L. Johnson, E.W. Andrews, B.J. Welch, The Impact of Anode Cover Control and Anode Assembly Design on Reduction Cell Performance, Proceedings of the Minerals, Metals \& Materials Society (TMS), TMS, Charlotte, NC, 2004, pp. 199-206.

[27] M.L. Slaugenhaupt, J.N. Bruggeman, G. Tarcy, N.R. Dando, Effect of Open Holes in the Crust on Gaseous Fluoride Evolution from Pots, Proceedings of the Minerals, Metals and Materials Society (TMS), TMS, San Diego, CA, 2003, pp. 199-204.

[28] J.N. Bruggemann, Pot Heat Balance Fundamentals, Proceedings of the 6th Australasian Al. Smelter Technology Conference and Workshops, 1998, pp. 167189.

[29] C.C. Wei, J.J.J. Chen, B.J. Welch, V.R. Voller, Modelling of dynamic ledge heat transfer, Proceedings of the Minerals, Metals and Materials Society (TMS), TMS, Orlando, FL, 1997, pp. 309-316.

[30] M.P. Taylor, The Influence of Process Dynamics on the Heat Balance and Cell Operation in the Electrowinning of Aluminium, Ph.D. thesis, University of Auckland, 1984.

[31] G. Vidalain, L. Gosselin, M. Lacroix, An Enhanced Thermal Conduction Model for the Prediction of Convection Dominated Solid-Liquid Phase Change, International J. Heat Mass Transfer 52 (2009) 1753-1760.

[32] V.R. Voller, C.R. Swaminathan, General Source-Based Method for Solidification Phase Change, Numer. Heat Transfer B 19 (1991) 175-189.

[33] C.R. Swaminathan, V.R. Voller, A General Enthalpy Method for Modeling Solidification Processes, Metall. Trans. B (1992) 651-664.

[34] V. Alexiades, A.D. Solomon, Mathematical Modeling of Melting and Freezing Processes, Hemisphere, Washington, DC, 1993, pp. 34-38.

[35] J.V. Beck, B. Blackwell, C.R. St.-Clair, Inverse Heat Conduction: Ill-Posed Problems, John Wiley \& Sons, New York, 1985.

[36] A.N. Tikhonov, V.Y. Arsenin, Solution of Ill-Posed Problems, Winston \& Sons, Washington, D.C., 1977.

[37] S.J. Julier, J.K. Ulhmann, H.F. Durrant-Whyte, A New Method for the Nonlinear Transformation of Means and Covariances in Filters and Estimators, IEEE Trans. Automat. Control 45 (2000) 477-482.

[38] W.H. Press, S.A. Teukolsky, W.T. Vetterling, B.P. Flannery, Numerical Recipes in $\mathrm{C}^{++}$, 2nd edition, Cambridge University Press, New York, 2002. 